FTUAM/96-13

\title{
Do classical configurations produce Confinement?
}

\author{
A. González-Arroyo and A. Montero \\ Departamento de Física Teórica C-XI, Universidad Autónoma de Madrid, \\ 28049 Madrid, Spain.
}

March 1996

\begin{abstract}
We show that certain classical $\mathrm{SU}(2)$ pure gauge configurations give rise to a non-zero string tension. We then investigate cooled configurations generated by Monte Carlo simulations on the lattice and find similar properties. We infer evidence in favour of a classical model of Confinement.
\end{abstract}




\section{Introduction}

Lattice calculations have given considerable evidence that QCD and YangMills theories are confining. In most cases, the reason for having a non-zero value of the string tension is hidden under a complicated, numerical computation. On the other hand, many authors have attempted understanding Confinement in a microscopic and qualitative way. Although it is unclear whether this is possible at all, it is certainly desirable, since it provides more physical insight into the origin of the behavior. In the early years of QCD, Polyakov [1] put forward the idea that classical configurations might play a crucial role in performing this goal. He showed, that pseudoparticle configurations (solutions of the euclidean equations of motion) are indeed very important in this respect in several theories. It soon became clear, however, that for QCD, the basic pseudoparticle (the instanton) cannot produce Confinement. Instantons seem, nonetheless, crucial in understanding other properties of the QCD vacuum [2, 3, 4. On the other hand, Callan, Dashen and Gross [5] proposed that other configurations - merons- $\left(\right.$ singular $Q=\frac{1}{2}$ configurations found by De Alfaro, Fubini and Furlan [6]) could indeed be responsible for this property.

Quite different approaches to the description of the QCD vacuum and Confinement were taken by other authors. The Copenhagen group ( for a review see [7] ) described the QCD vacuum as a liquid of magnetic flux tubes. A discrete version of magnetic flux tubes - fluxons - was also proposed as a mechanism for Confinement [8]. Fluxons are indeed the relevant disorder configurations that cause Confinement for the discrete $Z(N)$ gauge theories. For other gauge groups, one can define effective $Z(N)$ degrees of freedom and project out the dynamics for them. A different way to introduce $Z(N)$ degrees of freedom for a non-abelian gauge theory is through the topology of boundary conditions. It is remarkable that these twisted boundary conditions (TBC) [9] were found to be related to the configurations of the Copenhagen group.

The most widely accepted description of Confinement is based in the Dual-Superconductor picture [10, 11]. Confinement is seen as similar to Su- 
perconductivity, but with electric and magnetic fields exchanged. Hence, the condensation of a magnetically charged Higgs field leads to Confinement. The mechanism is seen to work nicely in compact QED [12 and in N=2 supersymmetric Yang-Mills [13]. For N=1 Yang-Mills theory, the whole thing is a bit more unclear. One has to explain what is the magnetic Higgs field (a priori no such field is present in the theory) and what is the mechanism that causes condensation: One needs the counterpart of the BCS theory. See Ref [14] for interesting ideas in this direction.

Recently, a new proposal for understanding Confinement in $\mathrm{SU}(2)$ pure gauge theory was put forward by our group [15, 16]. The basic idea of the proposal is that a class of quasi-self-dual classical configurations produces Confinement. These configurations are made of self-dual and anti-self-dual regions glued together. There is still considerable lack of understanding of how this gluing takes place, what is the typical size and shape of the regions, etc. The occurrence of self-dual or anti-self-dual pieces patched together resembles the instanton liquid model of Diakonov, Petrov and Shuryak [3, 国. However, our main point of departure from their picture is that, in our case, instantons loose their individuallity and dissolve into closely packed multiinstanton configurations. This introduces considerable difficulty into handling such configurations. Fortunately, we claim to have a good description of the main bulk of these configurations responsible for Confinement: it can be looked at as a liquid of $Q=\frac{1}{2}$ self-dual (or anti-s-d) lumps. This resembles enormously the picture of Ref. [5]. However, our configurations have, to the best of our knowledge, little to do with merons. The configurations we have in mind are smooth and emerge as deformations of a periodic array of $Q=\frac{1}{2}$ lumps known to exist [17] in the presence of twisted B.C.'s. We refer the reader to Ref. [16 for a more thorough discussion of this scenario. In particular, we showed there how a scale (mean separation between lumps, for example) is selected dynamically for the model. The approximate value of this scale was extracted from a semiclassical computation to be $0.7 \mathrm{fm}$, which was shown to give rise to values for the string tension and the topological susceptibility in rough agreement with measured ones.

To proceed further, one would like to check the main ingredients of the 
model: a) there are quasi-self-dual configurations which give rise to a non-zero string tension; b) these configurations are arranged into lumps of topological charge $|Q|=\frac{1}{2}$; c) the configurations that dominate the Yang-Mills vacuum are given by gaussian (and higher) fluctuations around these classical ones. In Ref. [20] we took advantage of the possibility of checking some of these points by studying the structure of lattice configurations. What we actually did, was to generate a collection of fairly uncorrelated SU(2) Yang-Mills configurations at $\beta=2.325$, on an $8^{3} \times 64$ lattice with twisted B.C.'s. Then, in order to get rid of the noise and inspired by the work of Ref. [18, we cooled these configurations with the method of Ref. [19] and $\epsilon=-0.3$ from 0 to 50 cooling steps. Finally, we analyzed the resulting cooled configurations to find local action density maxima which we called peaks. Our results were as follows. Indeed, the configurations which one gets after cooling are quasiself-dual and their total action divided by the number of peaks is close to $4 \pi^{2}$, as our model of $Q=\frac{1}{2}$ lumps predicts. Although we lack a general and neat method to discriminate which peak is an ordinary instanton ( which should also be present) and which is a $Q=\frac{1}{2}$ one, for low-densities we found a clearcut identification method which confirmed that the action per peak result is indeed due to the presence of a majority of $Q=\frac{1}{2}$ lumps.

To see the relation of these lumps with Confinement, we computed the string tension for our configurations. It turns out, that the effective string tension, measured from Polyakov loop correlations, decreases with the number of cooling steps. Our main observation was that the number of peaks also decreases, due to instanton-antiinstanton annihilation (which is known to be induced by cooling). Furthermore, there is a very strong correlation between both decreases: while the string tension varied by more than $40 \%$ from 20 to 50 cooling steps, the quantity $K \equiv \sigma / \sqrt{D}$ only varied by less than $2 \%$ ( $\sigma$ is the string tension and $D$ the density of peaks).

Indeed, we claim that the dimensionless variable $K$ is a fundamental quantity for all models of Confinement based on classical configurations. Since the classical Yang-Mills theory has no scale, if we expand the spatial size by some factor, this should affect densities and square string tension in the same way, but $K$ would not change. Using the mentioned estimated 
$(0.7 \mathrm{fm})^{-4}$ density and the value of $5 \mathrm{fm}^{-2}$ (taken as normalization) for the string tension, one gets $K \sim 2.5$, which was consistent with our numerical results.

There are some possible doubts or criticism on our previous work which we will now explain. The first one has to do with the use of TBC's. It could be argued, that the presence of $Q=\frac{1}{2}$ objects in our data is an artifact of the use of these B.C.'s, which stabilize these lumps. Our reply is that B.C's should not affect very much whenever the size is large enough to contain many lumps, which we claimed was the case. A different point of criticism, could come from our identification of the effective string tension, describing correlations at distances of 5-8 lattice spacings, with the asymptotic value which signals Confinement. In this respect the work of Teper [21] cast some doubt on the usefulness of cooling for investigating the structure of the vacuum. Teper argued that cooling, being a local algorithm, should leave the string tension unaltered. The decrease at fixed distance of the effective string tension was given no physical significance. Furthermore, he argued then that the true string tension had little to do with the classical structures revealed by cooling, and followed from long-wavelength fluctuations of a different nature. However, we can argue back, that the recovery of the uncooled string tension at much larger distances is not in contradiction with a classical picture, since after all, the basic effect induced by cooling (the annihilation of pairs) is also local in nature and should produce the same effect.

In this work, we are trying to obtain a stronger and more direct evidence supporting our argumentation.

\section{Data}

Are there some self-dual configurations which give rise to a non-zero string tension? To answer this question, we have produced such configurations and measured Wilson loops on them. In view of our theoretical prejudices, we

started with a periodic array of $Q=\frac{1}{2}$ instantons. This was obtained by taking the $4^{4}$ lattice (with twisted boundary conditions) single lump configuration and gluing it to itself to produce a $16^{3} \times 8$ (purely periodic B.C.) 
configuration. Due to the periodic arrangement of the resulting 128 lumps, this configuration is not suitable by itself. To randomize the position of the lumps, we applied a series of Monte Carlo steps to this configuration using Wilson action and several values of $\beta$ in the range $[3,4]$ in steps of 0.2 . The number of Monte Carlo steps was taken such that the final spatial distribution of peak locations was uniform within errors. For $\beta=3.0-3.2,50$ steps was found sufficient, while the number had to increase for larger $\beta$. Finally, to eliminate noise, we applied up to 50 cooling steps (in steps of 10) with the algorithm of Ref. [19] and $\epsilon=-0.3$. For each value of $\beta, 10$ different configurations were generated in the same way. The results are basically consistent in all cases, so we will illustrate them, by showing the numbers for $\beta=3.2$ and 50 Monte Carlo steps and $\beta=3.8$ and 75 Monte Carlo steps. After the first 10-20 cooling steps, things change very little, so we will quote only the results for 10 and 50 cooling steps. These are given in Table I.

The main characteristics of the data are the following. The configurations are close to self-dual, as can be seen by comparing the mean action divided by $8 \pi^{2}$ with the mean topological charge. The Monte Carlo steps have, not only randomized the position of the peaks, but also decreased their number down to the 20 to 40 range. However, in agreement with the initial configuration they all have positive topological charge and an action per peak which is close to $4 \pi^{2}$. This is also similar to the figure obtained for our TBC data [20]. Next, comes the crucial point: the string tension.

We measured the average value of $R \times T$ Wilson loops, for $R, T$ going from 1 to 7 in all the $16 \times 16$ planes ( 3 planes), over all the positions of the loops and the 10 independent configurations. From these averages we extracted the Creutz ratios $\chi(R, T)$. These numbers, show a dependence on the loop size $R \times T$ which flattens up for larger values. Since exploring larger loops was limited by our lattice sizes (and this by our computer memory) we tried to understand the main origin of the dependence. Creutz ratios are by definition insensitive to perimeter and constant terms in the exponent of Wilson loop averages. However, scale-invariant perturbative-like terms do not drop out and produce a $\gamma(1 /(R(R-1))+1 /(T(T-1)))$ dependence. We 
henceforth fitted our Creutz ratios data to the form:

$$
\chi(R, T)=\sigma-\gamma\left(\frac{1}{R(R-1)}+\frac{1}{T(T-1)}\right)
$$

The errors on $\chi(R, T)$ were obtained from the dispersion over the 10 configurations. The fits are excellent for all number of cooling steps. Fitting from $R, T=4$ onwards gives $\chi^{2}$ values which are less than one for 16 degrees of freedom and 2 parameters. Fitting from $R, T=3$ or 5 give also good $\chi^{2}$ values and consistent results. To show the quality of our results, we display in Fig. 1 those for $\beta=3.2$ and $50 \mathrm{MC}$ steps. What we actually plot is the combination

$$
\sigma(R, T) \equiv \chi(R, T)+\gamma\left(\frac{1}{R(R-1)}+\frac{1}{T(T-1)}\right)
$$

as a function of the area $R \times T$. The constancy of the quantity is quite impressive, showing that Creutz ratios can be very well described by an area law and a perturbative-like piece. From the plot it is also apparent that data of different sizes are strongly correlated (which explains the tiny $\chi^{2}$ values). Hence, the errors given by the fit are unrealistic. A safe estimate for the error of $\sigma$ is $10 \%$. The result is good for all number of cooling steps. It is also clear from Fig. 1 and Table I, that the value of the string tension $\sigma$ decreases with the number of cooling steps, although much less strongly than for our TBC data. This was to be expected, since in this case the basic positive-negative charge annihilation mechanism is almost absent. The resulting $\mathrm{K}$ values are not far from those obtained for the TBC's configurations.

Having established that certain configurations do produce a non-zero string tension, we proceed to the analysis of another crucial point for our argumentation: We have argued that the main conclusions of our previous paper [20] were not essentially biased by the use of twisted B.C.'s. For that purpose, we have Monte Carlo generated a total of 50 configurations on a $16^{3} \times 8$ lattice with periodic boundary conditions, using Wilson action and $\beta=2.325$. Out of them, 30 configurations were generated sequentially, separated by 500 heat bath sweeps, after some initial 5000 sweeps. Each one of the remaining 20, are obtained independently, by applying 500 Monte Carlo 
sweeps to an initial configuration, which was taken as fully ordered for 10 configurations and random for the remaining 10 . We have analyzed independently all three sets of configurations and found perfect compatibility, so we will henceforth consider the 50 configurations together. Finally, we applied up to 100 cooling steps (with $\epsilon=-0.3$ ) to them, and performed the same analysis to the cooled configurations as employed before. The results are summarized in Table II and Fig. 2.

In this case, both positive and negative topological charge peaks are present and, hence, the configurations are neither self-dual nor anti-self-dual. However, notice from Table II, that the integral of the absolute value of the topological charge density, labeled $\tilde{S}$, takes values close to those of the total action divided by $8 \pi^{2}$. This we interpret as a sign that the configuration is locally close to self-dual or anti-self-dual with regions connecting both areas. This is confirmed by the self (or anti-self)-duality of most peaks. Again, we find that the mean action per peak is close to the $4 \pi^{2}$ prediction of our model, and to the twisted B.C. results.

If we now look at the analysis of Wilson loops averages and Creutz ratios, we find similar results as before. Fits to Formula 1 are excellent as seen in Fig. 2, giving $\chi^{2}$ values per degree of freedom smaller than one. Now, the string tension decreases strongly with cooling, dropping by a factor 3.5 from 10 to 100 cooling steps. However, this variation is correlated with the drop in the number of peaks, giving a value of $K$ which changes only by $20 \%$. We also stress, that the actual value, close to 2.5 , is in agreement with our theoretical estimate, the result for TBC's [20] and our self-dual configurations (these need not give exactly the same value). We hence see that, despite the change in lattice size, boundary conditions, and the use of different observables to extract the string tension, the main features of our TBC results are nicely verified. Another interesting point is that, if we convert the string tension to physical units as in Ref. [20], we get for 10 cooling steps a value of $5.21 \mathrm{fm}^{-2}$, close to the uncooled infinite volume string tension, taken as normalization to $5 \mathrm{fm}^{-2}$. 


\section{Conclusions}

In summary, our results make it clear that the non-thermal self-dual configurations obtained from the array of $|Q|=\frac{1}{2}$ lumps do produce a non-zero value for the string tension. On the other hand, thermalized configurations generated at $\beta=2.325$ and periodic boundary conditions show a similar behavior with values of the quantity $K$ ( string tension divided by square root density) which are close to the previous ones, those found for the TBC data and the expectations of our model of the Yang-Mills vacuum. We comment that our results are quite economical in what regards computer resources. The self-dual data required 24 hours for each value of $\beta$ (10 configurations) in an HP-9000 730 workstation. The thermalized configurations required 250 hours ( for 50 configurations) in total.

We also want to mention that, in all cases, the mean action per peak is found to be close to the $4 \pi^{2}$ value predicted by the $|Q|=\frac{1}{2}$ liquid model of the vacuum Ref. [16]. In this case, however, we lack a method of identifying each peak, but it is quite unnatural to think that, given the general agreement in all other aspects, a similar value to the one found for the twisted boundary conditions results will have a different origin.

One of us (A.G-A) wants to thank D. Diakonov, V. Petrov, P. van Baal, J. Verbaarschot and the rest of participants in the 1995 Trento workshop on Non-perturbative approaches to QCD for useful conversations and the possibility to discuss all ideas at length. This work was supported by the CICYT grants AEN93-0693 and the EC network CHRX-CT93-0132.

\section{References}

[1] A. M. Polyakov, Phys. Lett. 59B (1975), 82.

[2] G. 't Hooft, Phys. Rev. Lett. 37 (1976), 8.

[3] D. I. Diakonov and V. Yu Petrov, Nucl. Phys. B245 (1984), 259.

[4] E. V. Shuryak, Nucl. Phys. B203 (1982), 93, 116. 
[5] R. G. Callan, R. Dashen and D. J. Gross, Phys. Lett. 66B (1977), 375.

[6] V. De Alfaro, S. Fubini and G. Furlan, Phys. Lett. 65B (1976), 163.

[7] P. Olesen, Phys. Scr. 23 (1981), 1000.

[8] G. Mack, Phys. Rev. Lett. 45 (1980), 1378.

[9] G. 't Hooft, Nucl. Phys. B153 (1979), 141.

[10] G. 't Hooft, In A. Zichichi, editor, High Energy Physics, Proceedings of the EPS International Conference. Editrici Compositori, 1976.

[11] S. Mandelstam, Phys. Rep. 23C (1976), 245.

[12] M.E. Peskin, Ann. of Phys. 113 (1978) 122.

[13] N.Seiberg and E.Witten, Nucl. Phys. B426 (1994), 19; N.Seiberg, Phys. Rev. D49 (1994), 6857.

[14] G. 't Hooft, Nucl. Phys. B190 (1981), 455; J. Smit And A. van der Sijs, Nucl. Phys. B426 (1994), 19.

[15] M. García Pérez, A. González-Arroyo and P. Martínez, Nucl. Phys. B (Proc. Suppl.) 34 (1994), 228.

[16] A. González-Arroyo and P. Martínez, Nucl. Phys. B459 (1996), 337.

[17] M. García Pérez, A. González-Arroyo and B. Soderberg, Phys. Lett. 235B (1990), 177; M. García Pérez and A. González-Arroyo, J. Phys. A 26 (1993), 2667.

[18] M. Campostrini et al, Phys. Lett. 225B (1989), 403.

[19] M. García Pérez, A. González-Arroyo, J. Snippe and P. van Baal, Nucl. Phys. B413 (1994), 535.

[20] A. González-Arroyo, P. Martínez and A. Montero, Phys. Lett. 359B (1995), 159.

[21] M. Teper, Nucl. Phys. B411 (1994), 855. 
Table 1: Results for the non-thermal configurations obtained after $50 \mathrm{MC}$ sweeps at $\beta=3.2$ (Conf. I) and 75 sweeps at $\beta=3.8$ (Conf. $I I$ ), and $N_{\text {cool }}$ cooling steps. We give the mean values of the action $S$ in $8 \pi^{2}$ units, the topological charge $Q$ and number of peaks $N_{p}$. The results of our fit to Creutz ratios for $R, T \geq 4(\gamma$ and the string tension $\sigma)$ are given. The quantity $K \equiv \sigma /(\text { peak density })^{\frac{1}{2}}$ is also shown.

\begin{tabular}{||c||c|c|c|c|c|c|c||}
\hline Conf. & $N_{\text {cool }}$ & $\left\langle\frac{S}{8 \pi^{2}}\right\rangle$ & $\langle Q\rangle$ & $\left\langle N_{p}\right\rangle$ & $\sigma$ & $\gamma$ & $\mathrm{K}$ \\
\hline \hline$I$ & 10 & $18.8(1.0)$ & $15.8(1.2)$ & $31.8(2.4)$ & 0.067 & 0.140 & 2.16 \\
\hline$I$ & 50 & $15.3(1.0)$ & $15.1(1.0)$ & $24.3(3.3)$ & 0.052 & 0.094 & 1.91 \\
\hline$I I$ & 10 & $16.2(0.8)$ & $14.5(0.8)$ & $24.2(1.8)$ & 0.056 & 0.105 & 2.05 \\
\hline$I I$ & 50 & $14.2(0.8)$ & $14.1(0.8)$ & $19.9(1.8)$ & 0.046 & 0.074 & 1.87 \\
\hline
\end{tabular}

Table 2: The same as Table I, but for the thermalized configurations at $\beta=2.325$. In this case we give $\tilde{S}$ ( the integral of the absolute value of the topological charge density) instead of $Q$.

\begin{tabular}{||c||c|c|c|c|c|c||}
\hline$N_{\text {cool }}$ & $\left\langle\frac{S}{8 \pi^{2}}\right\rangle$ & $\langle\tilde{S}\rangle$ & $\left\langle N_{p}\right\rangle$ & $\sigma$ & $\gamma$ & $\mathrm{K}$ \\
\hline \hline 10 & $35.1(0.6)$ & $22.7(0.5)$ & $77.8(1.1)$ & 0.124 & 0.272 & 2.54 \\
\hline 20 & $19.9(0.5)$ & $14.2(0.5)$ & $38.2(0.7)$ & 0.093 & 0.250 & 2.70 \\
\hline 40 & $11.8(0.5)$ & $9.2(0.5)$ & $19.5(0.7)$ & 0.063 & 0.185 & 2.58 \\
\hline 100 & $6.1(0.4)$ & $5.4(0.4)$ & $8.8(0.4)$ & 0.035 & 0.107 & 2.11 \\
\hline
\end{tabular}




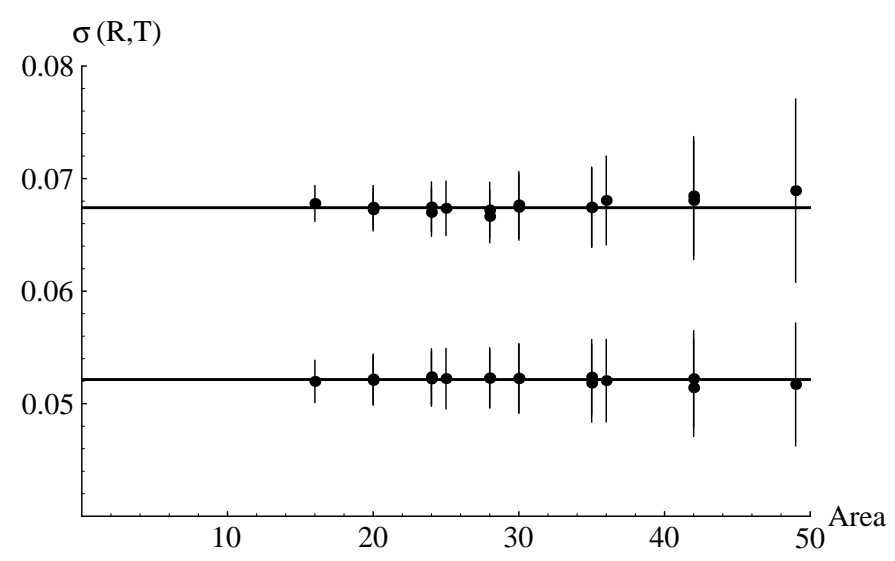

Figure 1: The lattice String Tension approximant $\sigma(R, T)$ (Formula 2) is plotted as a function of the Area $R \times T$ for the non-thermal configurations ( $\beta=3.2$ and $50 \mathrm{MC}$ steps) after 10 (upper points) and 50 (lower points) cooling steps. The horizontal lines are the best fit values given in Table I.

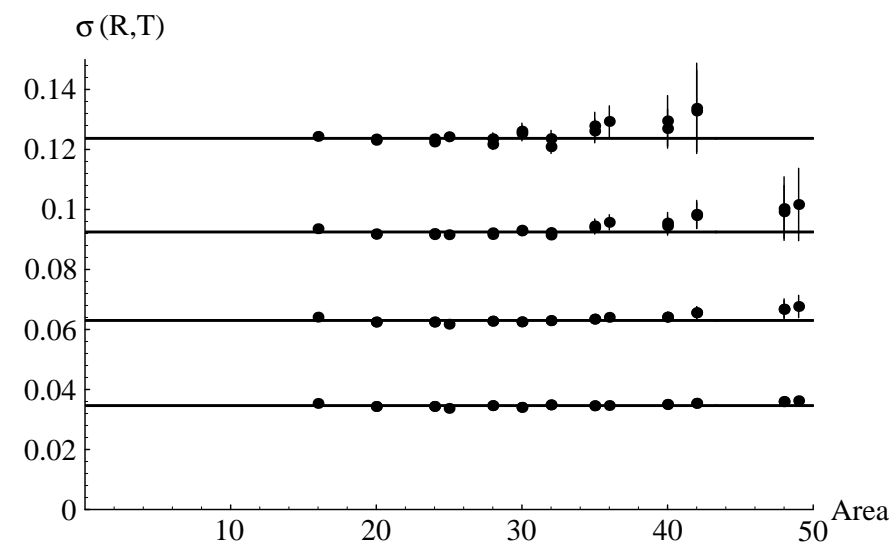

Figure 2: The same as Fig. 1 but for the thermalized data at $\beta=2.325$. From top to bottom the data for 10, 20, 40 and 100 cooling steps is displayed, together with the best fit horizontal lines. 